\title{
END EFFECTS IN INSTANTANEOUS TORSION OF INITIALLY STRETCHED ELASTOPLASTIC CYLINDERS*
}

\author{
$\mathrm{BY}$ \\ D. DURBAN' ${ }^{1}$ AND W. J. STRONGE \\ University of Cambridge, Cambridge, England
}

1. Introduction. The Saint-Venant theory for torsion of a slender cylinder has a cross-sectional distribution of shear stress that is a unique function of the material properties, stretch, and twist. When the stresses are a consequence of tangential tractions imposed on an end of the cylinder, any other statically equivalent distribution of tractions will induce a perturbation on the stress field near the end of the cylinder. Investigations of the decay of this perturbation in linear elastic cylinders date back to a 1906 paper by Pursar [1]. For any self-equilibrating tangential traction field imposed on an end of the cylinder, his eigenfunction expansion analysis predicts a nonoscillatory exponential decay of the perturbation with distance from the end. The eigenfunction with the smallest decay rate can be expressed as $\exp \left(-k_{1} z / R\right)$, where $z \geq 0$ is the axial coordinate, $R$ is the radius of the cylinder, and $k_{1}=5.13562$ is the first root for the Bessel function $J_{2}(k)=0$. This result is universally valid for all isotropic linear elastic solids.

The present paper considers the decay of a self-equilibrating torsional disturbance imposed at one end of an elastoplastic cylinder that is axially stretched. A set of selfequilibrating tangential tractions is suddenly imposed on the end of the stretched cylinder along with a uniform axial strain rate that maintains continuous plastic loading. The eigenfunctions for the rate boundary value problem associated with this loading are determined. The result is again a nonoscillatory exponential decay of the prescribed end perturbation. Unlike the linear elastic case, however, the characteristic decay length exhibits constitutive sensitivity. In some cases the decay length depends on the stretch.

2. Kinematics. Consider a semi-infinite circular cylinder, with current radius $R$, in a state of uniform axial stretch $a$ with associated tension stress $\sigma$. A polar coordinate system $(r, \theta, z)$ is located at the center of the end cross section so that the material occupies the domain $(R \geq r \geq 0, z \geq 0)$. The axially symmetric, instantaneous, torsion field imposed at $z=0$ is expected to activate only the circumferential velocity

\footnotetext{
*Received June 10, 1987.

${ }^{1}$ On sabbatical leave (until October 1987). Permanent address: Department of Aeronautical Engineering, Technion, Haifa 32000, Israel.
}

(C)1988 Brown University 
component $v=v(r, z)$ with the resulting shear strain rates

$$
\gamma_{r \theta}=\frac{1}{2}\left(v_{, r}-v / r\right), \quad \gamma_{\theta z}=\frac{1}{2} v_{, z}
$$

and spin components

$$
\omega_{r \theta}=-\omega_{\theta r}=\frac{1}{2}\left(v_{, r}+v / r\right), \quad \omega_{\theta z}=-\omega_{z \theta}=-\frac{1}{2} v_{, z}
$$

This field is accompanied by a decoupled uniform axial strain rate $\varepsilon_{z}=\varepsilon_{0}$ (along with the corresponding transverse contraction components), which is introduced to ensure continuous plastic loading (the shear stress rates associated with (1) do not produce plastic work).

3. Constitutive equations. Two finite strain versions of the isotropic hardening $J_{2}$ theory of plasticity will be adopted as constitutive models. First, we have the flow theory

$$
\stackrel{\nabla}{\boldsymbol{\sigma}}=2 \mu \mathbf{D}+\lambda \mathbf{I I} \cdot \mathbf{D}-2\left(\mu-\mu_{t}\right) \mathbf{N} \mathbf{N} \cdot \mathbf{D},
$$

where $\boldsymbol{\sigma}$ is the Cauchy stress tensor, $\underset{\sigma}{\sigma}$ its Jaumann rate, $\mathbf{D}$ the Eulerian strain rate, $\mathbf{I}$ the second-order unit tensor, and $\mathbf{N}=\mathbf{S} / \sqrt{\mathbf{S} \cdot \mathbf{S}}$ the unit normal tensor to the yield surface with $\mathbf{S}=\boldsymbol{\sigma}-(1 / 3) \mathbf{I I} \cdot \boldsymbol{\sigma}$ denoting the stress deviator; $\mu$ and $\lambda$ are the usual Lamé elastic constants, and $\mu_{t}$ is the tangent shear modulus defined by

$$
\frac{1}{\mu_{t}}=\frac{1}{\mu}+3\left(\frac{1}{E_{t}}-\frac{1}{E}\right)
$$

where $E_{t}$ is the tangent modulus and a known function (determined by the uniaxial tension test) of the effective stress $\sigma_{e}=\sqrt{(3 / 2) \mathbf{S} \cdot \mathbf{S}}$.

Specifying (3) for our problem we get the shear stress rates

$$
\stackrel{\nabla}{\tau}_{r \theta}=2 \mu \gamma_{r \theta}, \quad \stackrel{\nabla}{\tau}_{\theta z}=2 \mu \gamma_{\theta z},
$$

together with the uniform extension relations

$$
\sigma_{z}=E_{t} \varepsilon_{0}, \quad \varepsilon_{r}=\varepsilon_{\theta}=-\frac{1}{2}\left[1-(1-2 \nu) \frac{E_{t}}{E}\right] \varepsilon_{0},
$$

where $\nu$ is Poisson's ratio.

The second constitutive equation employed here is that of the deformation theory whose invariant form reads

$$
\boldsymbol{\sigma}=2 \mu_{S} \mathbf{e}_{L}+\lambda_{s} \mathbf{I I} \cdot \mathbf{e}_{L},
$$

where $\mathbf{e}_{L}$ is the finite logarithmic strain tensor (i.e., $\mathbf{e}_{L}=\ln \left(\mathbf{F} \cdot \mathbf{F}^{\mathrm{T}}\right)^{1 / 2}$ where $\mathbf{F}$ is the deformation gradient) and $\left(\mu_{s}, \lambda_{s}\right)$ are the secant Lamé-type moduli. Taking the Jaumann derivative of (7), we obtain the rate form

$$
\stackrel{\nabla}{\sigma}=2 \mu_{s} \mathbf{D}+\lambda_{s} \mathbf{I I} \cdot \mathbf{D}-2\left(\mu_{s}-\mu_{t}\right) \mathbf{N} \mathbf{N} \cdot \mathbf{D}+2 \mu_{s} \mathbf{Q} \cdot \mathbf{D},
$$

where the fourth-order tensor $\mathbf{Q}$ is determined by Hill's principal axes method [2]. When decomposed on the principal axes of strain, tensor $\mathbf{Q}$ has the nonvanishing components

$$
Q_{i j i j}=Q_{i j j i}=\frac{1}{2}\left[\frac{a_{i}^{2}+a_{j}^{2}}{a_{i}^{2}-a_{j}^{2}} \ln \frac{a_{i}}{a_{j}}-1\right] \text { no sum, for all } i \neq j,
$$


where $a_{i}$ are the principal stretches. For the circular cylinder in uniform axial stretch with $a_{z}=a$, we have from (7)

$$
a_{r}=a_{\theta}=a^{-1 / 2} \exp \left(\frac{1-2 \nu}{2} \bar{\sigma}\right) \quad \text { with } \bar{\sigma}=\frac{\sigma}{E} .
$$

It follows that the shear stress rates given by (8) are

$$
\stackrel{\nabla}{\tau}_{r \theta}=2 \mu_{s} \gamma_{r \theta}, \quad \stackrel{\nabla}{\tau}_{\theta z}=2 \mu_{s} \Lambda \gamma_{\theta z},
$$

where

$$
\Lambda=\frac{a^{3}+e^{(1-2 \nu) \bar{\sigma}}}{a^{3}-e^{(1-2 \nu) \bar{\sigma}}}\left[\ln \left(a^{3 / 2}\right)-\frac{1-2 \nu}{2} \bar{\sigma}\right] .
$$

The uniform extension relations of (8) remain identical with (6).

Thus, in contrast to the constant shear moduli of the flow theory (5), the shear moduli in (11) are deformation-dependent with $\mu_{s}$ decreasing (for common metals) as the initial tension increases. A useful relation in this context, similar to (4), is

$$
\frac{1}{\mu_{s}}=\frac{1}{\mu}+3\left(\frac{1}{E_{s}}-\frac{1}{E}\right)
$$

where $E_{s}$ is the secant modulus and a known function of the effective stress.

4. Equilibrium, boundary conditions, and solution. The rate form of the equilibrium equation is

$$
\boldsymbol{\nabla} \cdot(\stackrel{\nabla}{\boldsymbol{\sigma}}-\mathbf{D} \cdot \boldsymbol{\sigma}+\boldsymbol{\sigma} \cdot \mathbf{W}+\mathbf{I} \cdot \mathbf{D} \boldsymbol{\sigma})=\mathbf{0},
$$

where $\boldsymbol{\nabla}$ is the gradient operator and $\mathbf{W}$ the spin tensor. Recalling that the only active components of the stress rate tensor are $\stackrel{\nabla}{\tau}_{r \theta}$ and $\stackrel{\nabla}{\tau}_{\theta z}$, and using (1), (2), we find that (14) yields the single requirement

$$
\stackrel{\nabla}{\tau}_{r \theta, r}+\frac{2}{r} \stackrel{\nabla}{r \theta}_{r \theta}+\stackrel{\nabla}{\tau}_{\theta z, z}+\frac{\sigma}{2} v_{, z z}=0
$$

With the aid of the kinematic relations (1) and the stress rate-strain rate relations (5) or (11), we get from (15) the second-order differential equation

$$
v_{, r r}+\frac{1}{r} v_{, r}-\frac{v}{r^{2}}+c^{2} v_{, z z}=0,
$$

where for flow theory

$$
c^{2}=1+(1+\nu) \bar{\sigma}
$$

and for deformation theory

$$
c^{2}=\Lambda+\frac{\sigma}{2 \mu_{s}}=\frac{2 a^{3}}{a^{3}-e^{(1-2 \mu) \sigma}}\left[\ln \left(a^{3 / 2}\right)-\frac{1-2 \nu}{2} \bar{\sigma}\right] .
$$

A representative eigenfunction solution of (16) can be written as $v=$ $V(r) \exp (-k z / R)$ where $V(r)$ is the solution of

$$
V^{\prime \prime}+\frac{1}{r} V^{\prime}+\left(\frac{c^{2} k^{2}}{R^{2}}-\frac{1}{r^{2}}\right) V=0, \quad(\cdot)^{\prime}=\frac{d(\cdot)}{d r} .
$$


This equation is supplemented by the condition that the stress rate component $\stackrel{\nabla}{\tau}$ r should vanish at the boundary or, via the first of (1),

$$
v_{, r}-\frac{v}{r}=0 \quad \text { at } r=R
$$

Hence, the boundary condition for Eq. (19) becomes

$$
V^{\prime}-\frac{V}{r}=0 \text { at } r=R
$$

The solution of (19) and (21) is a linear combination of the first-order Bessel functions $J_{1}(\mathrm{ckr} / R)$ and $Y_{1}(\mathrm{ckr} / R)$. For the full cylinder the solution has to be bounded at $r=0$, so we are left with only the $J_{1}$ function. Compliance with the boundary condition (21) leads to the transcendental equation

$$
J_{2}(c k)=0,
$$

which is the same as Pursar's result except for the scale factor $c$. The proof that the eigenfunctions generated by (19) and (21), along with the associated eigenvalues from (22), produce self-equilibrating loads is straightforward and follows the standard proof of linear elasticity.

Of particular interest is the smallest positive root of (22), which governs the largest decay length of the end load,

$$
R / k=c R / k_{1}=c R / 5.13562 .
$$

The decay length for flow and deformation theories can be compared (Fig. 1) by using Eq. (23). With the flow theory, initial stretch has a negligible effect; usually $\bar{\sigma} \ll 1$ and so (17) becomes $c \simeq 1$ and $k=k_{1}$ for all practical purposes. On the other hand, with the deformation theory there is an appreciable dependence of $k$ on the stretch $a$. Using the approximation $\bar{\sigma} \ll 1$ in (18), we can write (23) as

$$
\frac{R}{k}=\left[\frac{a^{3} \ln \left(a^{3}\right)}{a^{3}-1}\right]^{1 / 2}\left(\frac{R}{k_{1}}\right)
$$

(this relation is exact for incompressible material where $\nu=\frac{1}{2}$ ). With the same material constants, the characteristic decay length in the deformation theory is larger than the decay length in the flow theory (Fig. 1). These results are, of course, applicable only as long as the tension $\sigma$ is below the uniaxial necking stress of the material.

It is interesting to note that a similar study [3] on end effects induced by axisymmetric self-equilibrating torsionless loads, imposed on the end of an initially stretched elastoplastic cylinder, has shown that flow theory predicts larger decay lengths than deformation theory. Furthermore, the decay length tends to increase without limit as the necking stress is approached [3]. Similar but slightly more complex behavior occurs in plane strain for the Ramberg-Osgood material [4]. These findings, which differ from the results of this paper, appear to reflect the complex nature of the validity of Saint-Venant's principle in finite strain plasticity. There is apparently a strong constitutive sensitivity coupled with the ground stress (stretch) field and the type of imposed self-equilibrating load. 


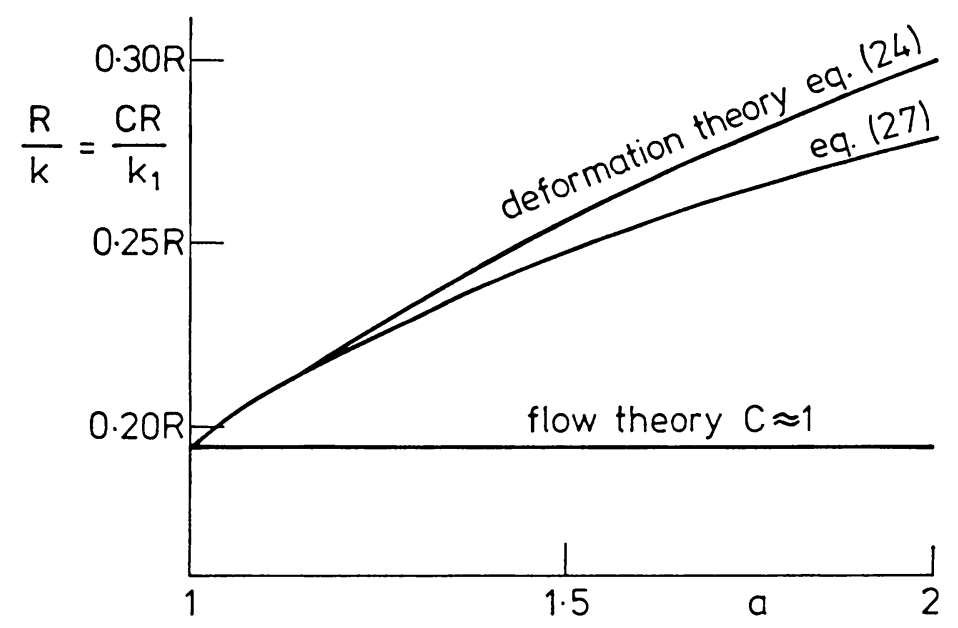

Fig. 1. The largest decay length for different constitutive relations as a function of initial stretch $a$

The present analysis can be extended to incrementally linear materials, where the shear relations are generalized by

$$
\stackrel{\nabla}{\tau}_{r \theta}=2 \mu_{1} \gamma_{r \theta}, \quad \stackrel{\nabla}{\tau}_{\theta z}=2 \mu_{2} \gamma_{\theta z},
$$

and the instantaneous moduli $\left(\mu_{1}, \mu_{2}\right)$ may depend on loading history. Proceeding as before, we arrive again at (23) with

$$
c=\left[\frac{2 \mu_{2}+\sigma}{2 \mu_{1}}\right]^{1 / 2} \text {. }
$$

Just to give an example, consider the $J_{2}$ incompressible elastoplastic solid proposed in [5] where $\mu_{1}=\mu_{2}=E_{s} / 3$. In this case Eq. (26) gives

$$
c=\left(1+\frac{3}{2} \ln a\right)^{1 / 2} \text {. }
$$

Again, this displays the constitutive sensitivity mentioned earlier (Fig. 1).

Finally, we note that the equivalent analysis for hollow tubes would result in an expression similar to (23) except that $k_{1}$ is the root of a more complicated transcendental equation involving both $J_{2}$ and $Y_{2}$. For thin-walled circular tubes with a deformed wall thickness $h$, the largest characteristic decay length is approximately $2 c h / \pi$.

Acknowledgment. One of us (D.D.) wishes to express his thanks for the kind hospitality of the Cambridge University Engineering Department. Part of this research was supported by the VPR-Aeronautical Engr. Research Fund, Technion, Haifa.

\section{REFERENCES}

[1] F. Pursar, Some applications of Bessel's functions to Physics, Proc. Roy. Irish Acad. Sec. A, 25-66 (1906)

[2] R. Hill, Aspects of invariance in solid mechanics, Advances in Appl. Mech. 18, 1-75 (1978)

[3] D. Durban and W. J. Stronge, On the validity of Saint-Venant's principle in finite strain plasticity, J. Appl. Mech. 110, 11-16 (1988) 
[4] D. Durban and W. J. Stronge, Diffusion of self-equilibrating end loads in plane strain plasticity, J. Mech. Phys. Solids 36, 459-476 (1988)

[5] S. Stören and J. R. Rice, Localised necking in thin sheets, J. Mech. Phys. Solids 23, 421-441 (1975) 